\title{
Patient involvement in selection of immunosuppressive regimen following transplantation
}

\author{
Joyce Popoola ${ }^{1,3}$ \\ Helen Greene ${ }^{2}$ \\ Margaret Kyegombe ${ }^{2}$ \\ lain A MacPhee ${ }^{1,3}$ \\ 'Department of Nephrology \\ and Transplantation, ${ }^{2}$ Department of \\ Pharmacy, St George's Healthcare \\ NHS Trust, ${ }^{3}$ St George's University \\ of London, London, UK
}

\author{
This article was published in the following Dove Press journal: \\ Patient Preference and Adherence \\ 12 December 2014 \\ Number of times this article has been viewed
}

Correspondence: Joyce Popoola Department of Nephrology and Transplantation, St George's Healthcare NHS Trust, Blackshaw Road, London, SWI7 0QT, UK

Tel +208725 2705

Fax +208725 2068

Email jpopoola@sgul.ac.uk
Abstract: Transplantation has made a considerable difference to the lives of many patients. However, feedback from patients indicates that although having a transplant is a hugely positive experience, having to take medications indefinitely is one of the biggest challenges. An ideal scenario would be no medications following a transplant. A compromise would be a minimal number of medications, with minimal restrictions and as simple a regimen as possible. Although there is considerable research going into fine-tuning the management of the immune response to a transplant, to date there is no universal regimen that enables patients to remain free of immunosuppressant medications, making adherence paramount to maintain long-term allograft survival. This paper reviews the available immunosuppressant regimens and factors influencing choice from both the clinician's and the patient's perspective. Factors influencing the decision-making process, such as quality of life for patients, their satisfaction, acceptability, and adherence uptake are reviewed. We conclude with a further assessment of patient choice as a factor in regimen selection, its impact on adherence, and its implications.

Keywords: adherence, transplant, allograft, immunosuppressants, patient involvement, satisfaction, decision-making

\section{Introduction}

Successful kidney transplantation is life-changing for those with organ failure. In the case of the heart, lungs, and liver, it is life-saving, as there are no long-term artificial replacements for these organs. Transplantation frees those with or approaching endstage renal failure from the impositions of restricted fluid intake, strict nutritional regimens, and the potential need for dialysis to a state of independence. Where an individual is a suitable candidate for a renal transplant, it provides them with a new lease of life and the ability to achieve near-normal kidney function, and freedom from attachment to machines and the significant time commitment of the actual process of performing the dialysis. In those requiring a pancreas transplant, it could mean freedom from repeated insulin injections, glucose monitoring, and dangerous hypoglycemic episodes, along with potential reversal of some of their diabetic complications.

It would of course be wrong to assume that transplantation is without its own attendant problems. Only patients fit enough to undergo major surgery can be transplanted. They also need to receive an appropriately matched organ in order to prevent loss of the transplant. The bane of the transplant recipient (patient) is the absolute necessity of continuous, appropriately timed, regular use of immunosuppression. Although matching across immunological barriers enables less immunosuppression in the peri-transplant period, some degree of immunosuppression is consistently necessary. 
The breakdown of this could lead to rejection, toxic damage to the transplant, and/or potential compromise to the recipient's health.

It is becoming vanishingly rare to lose transplanted organs in the first year in the absence of surgical or unsuspected immunological problems. Surgical techniques continue to improve and become more refined, and in addition, our understanding of the immunobiology around rejection is expanding. The long-term success of organs nowadays is dependent on appropriate long-term follow-up by individuals in a team with expertise and experience in the long-term management of post-transplant care. In the early days of transplantation, the surgeon who did the operation often led the post-transplant care. However, this care has evolved over time to encompass a multidisciplinary team that involves not only the transplanting surgeons but also transplant physicians with immunology training, specialist nurses, pharmacists to monitor adherence and drug interactions, dietitians to advise on changing diet, and psychologists and social workers to support individuals through the process. Additionally, the support of laboratory staff for chemistry, hematology, and immunology monitoring is required. Varying renal function and other chemistry, the immunosuppression used to overcome the natural immune response leading to issues around side effects and drug interactions, and the psychological and social impact of the process of receiving a foreign organ or tissue render the care of transplant recipients very complex.

Nonadherence is emerging as a major cause of transplant loss. It is an area that is not fully understood as, in many cases, there is no clear indication of the reason. It is also difficult to accurately assess, and tools are constantly being reviewed to try and aid assessment. At present this is either by direct methods, which can involve direct observation of patients taking medications or measurement of drug concentrations in blood or urine, or indirect methods. Indirect methods include physician estimation, diary methods, patient interviews, questionnaires, pill counts, and electronic monitoring.

\section{Review of available regimens and factors affecting choice of regimen}

Immunosuppression regimens for solid organ transplants are broadly divided into induction and maintenance regimens. The induction treatment consists of high-dose, more potent immunosuppressors in order to prevent the recipient's immune system rejecting the newly grafted organ immediately due to the sudden challenge to the immune system in the early transplant period.
The first attempts at immunosuppression used total body irradiation. Azathioprine was developed in 1957 and introduced by Sir Roy Calne in the 1960s, and accompanied by steroids. The addition of induction therapy using anti-thymocyte globulin or anti-lymphocyte globulin was introduced in the 1970s, giving about 50\% 1-year graft survival. The success of transplantation changed radically with the introduction of ciclosporin, the first calcineurin inhibitor, in the 1980s. Indeed, 1-year transplant survival went from $40 \%-50 \%$ to more than $80 \%$, with a significant reduction in mortality. ${ }^{1}$

The immunosuppressant protocols in most centers consisted almost uniformly of polyclonal antibody induction (or no induction antibody) and then maintenance immunosuppression with ciclosporin, azathioprine, and prednisolone. Development of new agents was driven by a desire to reduce side effects, such as infections and malignancy, as a result of excessive immunosuppression. Additionally, problems such as renal impairment, particularly noted in heart transplants where cardiac recipients often went on to require a kidney transplant.

Subsequent developments have included tacrolimus, a calcineurin inhibitor first shown to be associated with less rejection in liver transplants. Mycophenolate mofetil, a newer and more potent antiproliferative agent, is now frequently used in place of azathioprine. Sirolimus, an mTOR (mammalian target of rapamycin) inhibitor was introduced in 1999. It is currently used most commonly in patients unable to tolerate tacrolimus, but also increasingly in patients who have developed or who are at high risk of cancers. Induction therapy with biologic immunosuppressive agents has also broadened to include monoclonal antibodies to the IL-2 receptor, eg, basiliximab.

Development of additional immunosuppressant agents in the 1990s led to diversification of protocols. North America continued to favor the use of polyclonal antibodies for induction, while Europe was relatively prompt to switch to monoclonal antibody induction.

In the current decade, several of the North American centers have switched to using monoclonal antibodies for induction, including alemtuzumab (a humanized monoclonal antibody to CD52), mainly because of the increased incidence of lymphoma with anti-thymocyte and anti-lymphocyte globulin, particularly post-transplant lymphoproliferative disease. There have also been multiple studies showing equal efficacy with a relatively simpler regimen in relation to administration and a lower side effect profile. ${ }^{2,3}$ 
The more recent additions include costimulatory blockade, which was designed as a result of elegant research into understanding the signaling pathways involved in the $\mathrm{T}$ and $\mathrm{B}$ lymphocyte response. ${ }^{1}$ It was believed that adopting this approach would streamline the required immune suppression, thereby reducing other toxic side effects. Adoption of these agents has been variable, primarily due to the financial cost involved. Belatacept is a fusion protein containing a modified CD152 (CTLA-4) domain and a human IgG domain, which binds to the natural ligands for CD28, CD80, and CD86 molecules crucial for $\mathrm{T}$ lymphocyte costimulation. It was designed to provide prolonged graft survival while limiting the toxicity caused by calcineurin inhibitor-based regimens. It has the additional potential benefit of assisting adherence and is administered as a monthly intravenous infusion, with subcutaneous preparations that could be administered by patients themselves in development. Trials have shown better preservation of renal function than with calcineurin inhibitorbased regimens and a possible reduction in the development of donor-specific antibody, but this comes at the expense of a higher rate of episodes of rejection.

The increased range of immunosuppressive drugs now available has provided the opportunity for individualized agent choice and drug dosing. These include steroid avoidance or early steroid withdrawal, and similarly, calcineurin inhibitor minimization or withdrawal. More recently, some clinicians have adopted strategies of calcineurin inhibitor avoidance, with variable outcomes.

The majority of centers now aim to use dual therapy for longer-term graft maintenance. Commonly, this comprises the combination of a calcineurin inhibitor and an antiproliferative agent or low-dose steroids. In patients with malignancy or calcineurin inhibitor-related renal damage, calcineurin inhibitors replacement with mTOR inhibitors (sirolimus or everolimus) is increasingly being adopted. A few centers have been trialing long-term use of a single immunosuppressant agent. This is being achieved by using alemtuzumab, the costimulatory monoclonal antibody directed against CD52, which depletes both $\mathrm{T}$ and B lymphocytes. ${ }^{4}$

The increasing choices in immunosuppressant medications has led to considerable diversification in regimens used around the world, intercenter and indeed in some cases intracenter. This has the potential for making direct comparison of outcome data a challenge, and this is particularly an issue for randomized trials because they will never be able to compare all the available options. There remains a clear need for more effective, easily administered, and less toxic immunosuppressive drugs.

\section{Efficacy studies including comparative safety, and tolerability studies}

The advent of calcineurin inhibition (ciclosporin and later tacrolimus) was the major breakthrough in enabling successful transplantation. Unfortunately, these agents have attendant side effects, such as hypertension, toxicity to the grafts, diabetes, malignancy, and neurological adverse events. Studies looking at minimization of calcineurin inhibition, withdrawal, and avoidance protocols, particularly after kidney transplants, have had variable results.

More recently, the role of pharmacogenetics in early and appropriate dosing of calcineurin inhibitors for transplant recipients has been investigated. The most widely explored and the nearest gene to introduction into routine clinical practice to assist tacrolimus dosing is cytochrome P450 $3 A 5$ genotype. ${ }^{5,6}$

Attempts at reducing the side effect profile have led to various strategies in new protocols. With good short-term outcomes, effort has been concentrated on developing protocols to minimize toxicity and improve long-term graft survival. One of the most influential studies recently has been the SYMPHONY study, which has led to the widespread adoption of an immunosuppressive regimen based on lowdose tacrolimus, mycophenolate mofetil, and steroids with or without use of an anti-interleukin-2 receptor antibody for induction. ${ }^{3}$

\section{Importance of adherence to long-term transplant outcomes}

It is not surprising that poor adherence to immunosuppressive drug treatment is associated with a poor transplant outcome. In a meta-analysis of ten studies, nonadherence was found to increase the risk of graft failure by seven-fold when compared with adherent patients. ${ }^{7}$ Recently published data on the cause of renal transplant failure have indicated the key role of chronic antibody-mediated rejection associated with poor adherence to treatment. ${ }^{1}$ This has moved adherence up the agenda of aspects of transplant care that may be amenable to intervention to improve long-term transplant survival.

\section{Patient-focused perspectives: quality of life, satisfaction/acceptability, adherence, and uptake}

The health belief model offers a possible explanation for why transplant recipients adhere or not to immunosuppressant medications. The health belief model is a psychological health behavior change model, which was originally developed to help 
explain and predict the health-related behavior of patients in relation to uptake of health services in general. It suggests that individuals will take action to manage disease, such as adhering to medications, if they: perceive themselves as susceptible to a disease; believe the disease to have serious consequences; and believe the benefits of taking action to manage the disease outweigh the barriers to or costs of the action. ${ }^{8}$

The model suggests that the likelihood of taking action is affected by perceived barriers to action, perceived benefits of action, external influences that may cue action, and other variables, such as demographics, psychosocial factors, oncedaily dosing versus multiple daily doses, side effect profile, and cost for those patients who pay for their medications. ${ }^{8,9}$

Men, older patients, single young adults, and patients who have had transplants for a long time are traditionally described as being likely to be more nonadherent than others, ${ }^{9,10}$ although this has been called into question by other researchers in meta-analysis. ${ }^{11}$ Estimating the true degree of nonadherence among transplant recipients is not easy given that it often only becomes recognized after a measurable event. There is, however, also a significant proportion of patients who have subclinical nonadherence.

Most patients who do not adhere report forgetting doses, missing doses, being careless about doses, but less commonly report stopping immunosuppressant medications altogether for periods at a time and even more rarely stopping them altogether. Problems that are a direct result of nonadherence to immunosuppressant medications include graft rejection, decreased quality of life, increased health care costs, need for dialysis, morbidity, and mortality. ${ }^{6}$

It therefore becomes important to identify areas where health care professionals can potentially have a significant impact on adherence in their transplant recipients. Nonadherent organ recipients perceive greater barriers to medication adherence, are less likely to believe that their immunosuppressant medications are necessary, and are more likely to have concerns regarding them. In our anecdotal experience, a recent issue that has led to patients believing that they do not need immunosuppressive drugs is the publication of data on a small cohort of immunologically tolerant transplant patients who stopped immunosuppressive treatment and have preserved graft function. ${ }^{12}$ Unfortunately, the nonexpert reader may not be able to put these data into an appropriate perspective and arrive at an erroneous conclusion.

Nonadherent recipients also have a tendency to report lower life satisfaction than adherent recipients. Thus, strategies for eliminating barriers to adherence, as well as identifying and alleviating patient concerns about immunosuppressant medications, are potentially promising areas of focus for future adherence programs. By implementing successful adherence interventions, health care professionals may be able to enhance not only adherence of transplant patients to essential immunosuppressant medications, but also their health status and life satisfaction, a key factor associated with quality of life. ${ }^{13}$

\section{Patient choice as a factor in regimen selection and impact on adherence}

Involving patients in health care decisions can make a potentially significant and enduring difference to health care outcomes. ${ }^{14-16}$ The ethical need for patient choice, respecting autonomy, and responding to patients' demands for more involvement in their treatment is becoming widely recognized. ${ }^{17-19}$

Pharmaceutical interventions offer well documented benefits at the population level. However, for the individual patient, the immediate clinical effect may be side effects, whereas benefits may not be possible to identify or measure directly. ${ }^{20}$ This is why patient choice can have a significant impact on adherence.

Advances in immunosuppressant medications have resulted in notable improvements in post-transplant outcomes, particularly in the first year. However, longer-term outcomes have not improved to the same extent. ${ }^{21}$ One of the major factors contributing to the incidence of late acute rejection and graft loss in transplant patients is nonadherence. ${ }^{22}$ The prevalence of nonadherence to medication following kidney transplantation has been reported to be in the order of $15 \%-55 \%$. $^{7,23}$

It is now understood that problems with adherence can affect all transplant recipients and are not limited to traditional "risk groups", such as the elderly and adolescents. In a large meta-analysis including studies of kidney, heart, liver, and pancreas transplants, nonadherence was not shown to be associated with sex, age, ethnicity, or level of education. ${ }^{11}$ There can be many other confounding factors in the study of adherence, such as age, income, time since transplant, and form of immunosuppressant prescribed. ${ }^{24}$ Post-transplant nonadherence is increasingly regarded as a preventable cause of late acute rejection, chronic rejection, and graft loss. ${ }^{7,22}$ Since medication adherence is usually not predictable, emphasis should be placed on increasing adherence in all patient groups. ${ }^{13,25}$ 
Addressing medication nonadherence often requires a multifaceted approach, which includes developing methods of identifying nonadherence, models to identify determinants of and factors promoting nonadherence, and strategies to educate patients concerning their therapy. Finally, practical interventions by multidisciplinary practitioners need to be adopted. ${ }^{26}$

Shared decision-making allowing patient choice, as opposed to clinicians merely making decisions on behalf of patients, is gaining increasing prominence in health care policy. ${ }^{27-30}$ Shared decision-making has been defined as:

An approach where clinicians and patients share the best available evidence when faced with the task of making decisions, and where patients are supported to consider options, to achieve informed preferences. ${ }^{14}$

This approach may be beneficial in managing adherence to immunosuppressive regimens.

Effective communication can enhance knowledge, involvement in decisions about interventions, autonomy, and empowerment of patients..$^{20}$ In contrast, poor communication can lead to anxiety, lack of confidence in the clinician, and to decisions based on misinterpreted information and inadequate communication. ${ }^{31}$ The prescriber should try to understand the health beliefs and expectations of the patient regarding their treatment. Adherence to therapy is more likely if the patient understands the reason for taking a drug and is involved in the decision to prescribe. Patients are more likely to have confidence in the prescriber if given basic knowledge of potential adverse effects and advice on what to do if they occur. They should be made aware of how to take the drug and for how long they will need to take it. ${ }^{32}$

Shared decision-making is supported by evidence from 86 randomized trials showing knowledge gained by patients, more confidence in decisions, more active patient involvement, and in many situations, informed patients elect for more conservative treatment options. ${ }^{33}$ However, the trend to allow more patient choice has led to concern regarding potential "abandonment", where clinicians offer information about treatment choice but no guidance. ${ }^{34}$ Indeed, doubts have been expressed by some health care professionals, suggesting that patients do not want to be involved in decisions, lack capacity or ability, might make "bad" decisions, or worry that shared decision-making is not practical, given constraints such as time pressure.

Others claim that shared decision making is already in place, but data from patient experience surveys indicate that this is not generally the case. ${ }^{35,36}$ Therefore, it is essential that in order to advocate the uptake of shared decision-making, both clinicians and patients need to support the underlying rationale. There are a number of challenges that clinicians will face. These include low health literacy or low numeracy, in addition to cultural backgrounds that may lack a tradition of individuals making autonomous decisions. It is prudent that the shared decision-making process is built on core skills of good clinical communication, with building a rapport and structured consultations. ${ }^{37}$

Shared decision-making involves three key steps, ie, "choice talk", "option talk", and "decision talk", where the clinician supports deliberation throughout the process. Choice talk refers to the step of making sure that the patient knows that reasonable options are available. Option talk refers to providing more detailed information about options, and decision talk refers to supporting the work of considering preferences and deciding on the best option. ${ }^{38}$

"Paternalism" has acquired a negative connotation in medicine, but has an inevitable role in the prescription of treatments with a complex basis. Ultimately, the complexity of decision-making around immunosuppressive treatment will challenge many patients with a need for substantial support based on the clinician's interpretation of the risks and benefits of treatment.

Patients will need time to interpret the information given to them and to consider personal preferences, particularly where outcome states are unknown. Deliberation may, in part, be done outside the clinical encounter, although patients often wish to consolidate their views with a clinician. ${ }^{38}$ Patients will want to discuss options with others, and Rapley has referred to this need as a "distributed deliberation process" ${ }^{39}$ Recognizing this need and allowing time for it is a cornerstone for effective shared decision-making. Decision support interventions, which summarize information in formats that are accessible to patients, using the most up-to-date evidence about harms and benefits can also be used ${ }^{40}$ These can be in concise formats, such as in brief text or diagrams, or can be extensive, as in booklets, websites, videos, and DVD, to be used by patients, their friends, and their families during and outside the clinic consultations. ${ }^{38}$

In summary, shared decision-making, enabling patient choice, may enhance adherence to immunosuppressive regimens. Clinicians need to be respectful and remain alert to the patient's individual preferences. Although challenges exist, the benefits of patient choice and involvement, and the skills required to achieve this approach, need to be given much higher priority. 


\section{Impact of choice of immunosuppressive drug regimen}

The drugs in widespread use for immunosuppressive treatment in transplant recipients have all now come off patent, with the recent availability of generic preparations at vastly reduced cost compared with the innovator products. In health care systems where the patient has to buy their own medication, use of cheaper generic products may have a major impact on adherence. It is of note that the reported incidence of nonadherence in renal transplant recipients is much higher in the USA, where state provision of immunosuppressive treatment is limited to 3 years (currently under review) than in health care systems such as the UK and Canada which have long-term state provision of treatment. ${ }^{41}$ There is major pressure from state and insurance funders to use generic rather than innovator immunosuppressive agents to save money. Any patient being changed from treatment with an innovator to a generic drug ought to be involved in the decision-making process in order to maintain trust. There tends to be understandable suspicion, which can be overcome by provision of evidence of equivalence, and it is important that clinicians are transparent as to the underlying reason for change. This is a situation where the wishes of the individual patient may be in conflict with the needs of the health care system. The decision-making process therefore goes beyond just the patient and their health care providers in a state-funded health care system; for instance, in the UK, the regulatory body National Institute for Health and Care Excellence. ${ }^{42}$

There are some data indicating a very small but statistically significant increase in adherence by patients treated with once-daily rather than twice-daily tacrolimus. ${ }^{43,44}$ Patients who maintain the number of skipped doses after conversion from twice-daily to once-daily dosing might be better served by a twice-daily regimen. ${ }^{44} \mathrm{~A}$ potential barrier to this option is the increased cost of the once-daily preparation, where there is no generic option, which may actually impact negatively on adherence in health care systems where patients need to buy their own medications.

\section{Conclusion and implications}

It is clear that while transplantation can be life-saving or life-enhancing, maintaining the organ can be a challenge as a result of the necessity for lifelong immunosuppressants. Current knowledge and medicine do not allow for indefinite survival of grafts without long-term immunosuppression. In view of this, the clinician requires a broad knowledge and appropriate skills in the management of immunosuppression in order to enhance adherence and minimize side effects in the individual patient by tailoring their medication according to lifestyle, comorbidities, and immune status. It is clear that patient engagement is needed to achieve optimal adherence and ultimately maximal graft survival. Early identification of potential barriers and promoters of adherence is needed in the run-up to transplantation as well as in the follow-up period after transplant. In some instances, this may require a skill mix beyond the reach of the nurse, pharmacist, physician, or surgeon (see Table 1).

Adherence may be achieved as a result of guilt (eg, wanting to please family or the medical team), concern at being judged/marginalized although it is best sustained when self-motivated. Early identification of potential problems in relation to a patient's status and support network could have a significant influence on adherence. Understanding of the dynamics of a patient's social network can prove invaluable and could lead to identification of a couple of buddies for the organ recipient to support them through the process. Issues with forgetting medications, confusion around taking medicines at different times of the day, timing with food, and difficulty following instructions can often be circumvented with appropriate aids and techniques, such as alarms, drug cards, dosette boxes, and individualized education sessions.

In contrast, patients skipping medications to avoid side effects require an astute, supportive clinician and pharmacy involvement. Cycles of breakdown in adherence due to patients becoming fed up with regimens may require a major pattern change and input from a psychologist to work through precipitating factors and to develop motivating and alternative behavioral strategies. Beyond psychology input, psychiatric input may be required, and recognizing this requirement early is paramount, given that depression is commonly seen in patients with chronic disease and frequently presents as nonadherence. It is noteworthy that cries for help and suicide can also be played out through nonadherence.

It is becoming increasingly clear that organ transplantation goes beyond performing an operation and handing out pills. It is fair to say that solving adherence problems and developing solutions warrants focused research. There have been amazing breakthroughs achieved in complex surgical techniques in the past century. There is now scientific and medical understanding to overcome even the most challenging of immunological barriers to enable acceptance and function of a transplanted organ. Nonadherence is emerging as a major factor leading to graft loss. It should be viewed as a potentially modifiable factor influencing graft survival. However, ultimately, long-term survival of a transplant requires 
Table I Barriers, causes, and calls to action to improve adherence

\begin{tabular}{|c|c|c|}
\hline $\begin{array}{l}\text { Potential } \\
\text { adherence barriers }\end{array}$ & $\begin{array}{l}\text { Potential causes of } \\
\text { adherence barriers }\end{array}$ & Calls to action to overcome adherence barriers \\
\hline Medical & $\begin{array}{l}\text { Side effects } \\
\text { Drug interactions } \\
\text { Pill burden }\end{array}$ & $\begin{array}{l}\text { Involvement of pharmaceutical companies in modification of current } \\
\text { medications and development of new ones to reduce side effects } \\
\text { and interactions } \\
\text { Involvement of pharmacists and specialist nurses as well as physicians } \\
\text { to identify patients with side effects and potential drug interactions } \\
\text { Careful monitoring of drug levels to avoid toxic side effects or problems due } \\
\text { to inadequate dosing } \\
\text { Dietitian involvement to ensure appropriate medication delivery } \\
\text { and to ensure a healthy diet with only necessary restrictions } \\
\text { Regular review of entire patient prescriptions to rationalize pill burden } \\
\text { Ongoing liaison with community doctors and pharmacists }\end{array}$ \\
\hline Social & $\begin{array}{l}\text { Peer pressure } \\
\text { Restriction by work, social life } \\
\text { Disruption of routines, eg, job } \\
\text { loss, moving house, divorce, } \\
\text { transitioning to new units or } \\
\text { from pediatric to adult units }\end{array}$ & $\begin{array}{l}\text { Create awareness of transplantation and immunosuppression adherence } \\
\text { in society through educational sessions, social media, and television } \\
\text { Development of buddy programs to convert peer pressure } \\
\text { to positive support } \\
\text { Develop supportive pathways for seamless transition of young adults from } \\
\text { pediatric to adult services } \\
\text { Social worker involvement } \\
\text { Psychology services }\end{array}$ \\
\hline Emotional and mental & $\begin{array}{l}\text { Depression } \\
\text { Mood swings } \\
\text { Psychosis }\end{array}$ & $\begin{array}{l}\text { Early identification of symptoms in patients } \\
\text { Identification and involvement of patient's network (next of kin, family, } \\
\text { friends) where appropriate } \\
\text { Integrated support from psychologists and/or psychiatrists depending } \\
\text { on diagnosis }\end{array}$ \\
\hline Educational & $\begin{array}{l}\text { Illiteracy } \\
\text { Complex regimens }\end{array}$ & $\begin{array}{l}\text { Development of clear educational strategies to suit the patient's } \\
\text { educational level } \\
\text { Early recognition of patients who may struggle, ideally in the pre-transplant } \\
\text { period } \\
\text { Use of dosette boxes, blister packs, direct observation, alarms, and drug cards } \\
\text { Simplification of the immunosuppressant regimens, eg, use of once daily } \\
\text { regimens } \\
\text { Tailoring regimens to suit patient's lifestyle }\end{array}$ \\
\hline Financial & $\begin{array}{l}\text { Cost of immunosuppressant } \\
\text { medications }\end{array}$ & $\begin{array}{l}\text { Call to pharmaceutical companies to rationalize prices with potential } \\
\text { discounts for developing countries } \\
\text { Use of properly tested generics to create a competitive market } \\
\text { Government legislation to ensure support for immunosuppressant regimen } \\
\text { cost for patients without adequate insurance, subsidizing or covering cost } \\
\text { for the life of the transplant } \\
\text { Create awareness in patients about prioritization of their immunosuppressants } \\
\text { in situations of limited finances } \\
\text { Input from social workers }\end{array}$ \\
\hline
\end{tabular}

Note: The information in this table is not exhaustive but rather seeks to compartmentalize some of the potential barriers to adherence and offer potential solutions.

the uncompromised input of the patient, their support network, and the entire multidisciplinary clinical team.

\section{Disclosure}

The authors report no conflicts of interest in this work.

\section{References}

1. Halloran PF. Immunosuppressive drugs for kidney transplantation. N Engl J Med. 2004;351:2715-2729.

2. Hanaway MJ, Woodle ES, Mulgaonkar S, et al. Alemtuzumab induction in renal transplantation. N Engl J Med. 2011;364:1909-1919.
3. Ekberg H, Tedesco-Silva H, Demirbas A, et al. Reduced exposure to calcineurin inhibitors in renal transplantation. $N$ Engl $J$ Med. 2007;357:2562-2575

4. Chan K, Taube D, Roufosse C, et al. Kidney transplantation with minimized maintenance: alemtuzumab induction with tacrolimus monotherapy an open label, randomized trial. Transplantation. 2011;92:774-780.

5. Thervet E, Loriot MA, Barbier S, et al. Optimization of initial tacrolimus dose using pharmacogenetic testing. Clin Pharmacol Ther. 2010;87:721-726.

6. MacPhee IA, Fredericks S, Tai T, et al. Tacrolimus pharmacogenetics: polymorphisms associated with expression of cytochrome P4503A5 and P-glycoprotein correlate with dose requirement. Transplantation. 2002;74:1486-1489. 
7. Butler JA, Roderick P, Mullee M, Mason JC, Peveler RC. Frequency and impact of non-adherence to immunosuppressants after renal transplantation: a systematic review. Transplantation. 2004;77:769-776.

8. Janz NK, Champion VL, Stetcher VJ. The health belief model. In: Glanz K, Rimer BK, Lewis FM, editors. Health Behavior and Health Education. 3rd ed. San Francisco, CA, USA: Josey-Bass; 2002.

9. Chisholm-Burns M, Pinsky B, Parker G, et al. Factors related to immunosuppressant medication adherence in renal transplant recipients. Clin Transplant. 2012;26:706-713.

10. Chisholm MA, Kwong WJ, Spivey CA. Associations of characteristics of renal transplant recipients with clinicians' perceptions of adherence to immunosuppressant therapy. Transplantation. 2005;84:1145-1150.

11. Dew MA, Dimartini AF, De Vito DA, et al. Rates and risk factors for non-adherence to the medical regimen after adult solid organ transplantation. Transplantation. 2007;83:858-873.

12. Newell KA, Asare A, Kirk AD, et al. Identification of a B cell signature associated with renal transplant tolerance in humans. J Clin Invest. 2010; 120:1836-1847.

13. Jindal RM, Neff RT, Abbott KC, Hurst FP, Elster EA, Falta EM. Associations between depression and nonadherence in recipients of kidney transplants: analysis of the United States renal data system. Transplant Proc. 2009;41:3662-3666.

14. Stewart M. Studies of health outcomes and patient-centered communication. In: Stewart M, Brown JB, Weston WW, et al, editors. PatientCentered Medicine: Transforming the Clinical Method. Thousand Oaks, CA, USA: Sage Publications; 1995.

15. Savage R, Armstrong D. Effect of a general practitioner's consulting style on patient satisfaction: a controlled study. BMJ. 1990;301:968-970.

16. Kinmonth AL, Woodcock A, Griffin S, et al. Randomised controlled trial of patient-centred care of diabetes in general practice: impact on current wellbeing and future disease risk. BMJ. 1998;317:1202-1208.

17. Richards T. Partnership with patients. BMJ. 1998;316:85-86.

18. Coulter A, Entwistle V, Gilbert D. Sharing decisions with patients: is the information good enough? BMJ. 1999;318:318-322.

19. Coulter A. Paternalism or partnership? BMJ. 1999;319:719-720.

20. Edwards A, Hood K, Matthews E, et al. The effectiveness of one-to-one risk communication interventions in health care: a systematic review. Med Decis Making. 2000;20:290-297.

21. Meier-Kriesche HU, Schold JD, Kaplan B. Long-term renal allograft survival: have we made significant progress or is it time to rethink our analytic and therapeutic strategies? Am J Transplant. 2004;4: 1289-1295.

22. Denhaerynck K, Dobbels F, Cleemput I, et al. Prevalence, consequences, and determinants of non-adherence in adult renal transplant patients: a literature review. Transpl Int. 2005;18:1121-1133.

23. Vasquez EM, Tanzi M, Benedetti E, Pollak R. Medication noncompliance after kidney transplantation. Am J Health Syst Pharm. 2003;60: 266-269.

24. Chisholm MA. Issues of adherence to immunosuppressant therapy after solid-organ transplantation. Drugs. 2002;62:567-575.

25. Chisholm MA, Lance CE, Mulloy LL. Patient factors associated with adherence to immunosuppressant therapy in renal transplant recipients. Am J Health Syst Pharm. 2005;62:1775-1781.

26. Chisholm MA. Enhancing transplant patients' adherence to medication therapy. Clin Transplant. 2002;16:30-38.
27. O'Connor A, Wennberg JE, Legare F, et al. Toward the "tipping point": decision aids and informed patient choice. Health Aff (Millwood). 2007; 26:716-725.

28. Elwyn G, Coulter A, Laitner S, Walker E, Watson P, Thomson R. Implementing shared decision making in the NHS. BMJ. 2010;341:c5146.

29. Department of Health. Equity and excellence: liberating the NHS. London, UK: 2010. Available from: https://www.gov.uk/government/ uploads/system/uploads/attachment_data/file/213823/dh_117794.pdf. Accessed September 3, 2014.

30. Senate and House of Representatives. Patient Protection and Affordable Care Act. HR 3590. Washington, DC, USA: 2010. Available from: https://www.govtrack.us/congress/bills/111/hr3590/text. Accessed September 3, 2014.

31. Harmsen CG, Jarbøl DE, Nexøe J, et al. Impact of effectiveness information format on patient choice of therapy and satisfaction with decisions about chronic disease medication: the "Influence of intervention Methodologies on Patient Choice of Therapy (IMPACT)" cluster-randomised trial in general practice. BMC Health Serv Res. 2013;13:76.

32. Waller DG. Rational prescribing: the principles of drug selection and assessment of efficacy. Clin Med. 2005;5:26-28.

33. Stacey D, Bennett C, Barry M, et al. Decision aids for people facing health treatment or screening decisions. Cochrane Database Syst Rev. 2011;10:CD001431.

34. Quill TE, Cassel CK. Nonabandonment: a central obligation for physicians. Ann Intern Med. 1995;122:368-374.

35. Zikmund-Fisher BJ, Couper MP, Singer E, et al. The DECISIONS study: a nationwide survey of United States adults regarding 9 common medical decisions. Med Decis Making. 2010;30(5 Suppl):20S-34S.

36. Coulter A. Do patients want a choice and does it work? BMJ. 2010;341: 973-975.

37. Silverman J, Kurtz S, Draper J, et al. Skills for Communicating with Patients. Abingdon, UK: Radcliffe Medical Press; 1998.

38. Elwyn G, Frosch D, Thomson R, et al. Shared decision making: a model for clinical practice. J Gen Intern Med. 2012;27:1361-1367.

39. Rapley T. Distributed decision making: the anatomy of decisions-inaction. Sociol Health Int. 2008;30:429-444.

40. O'Connor A, Graham I, Visser A. Implementing shared decision making in diverse health care systems: the role of patient decision aids. Patient Educ Counsel. 2005;57:247-249.

41. Gill JS, Tonelli M. Penny wise, pound foolish? Coverage limits on immunosuppression after kidney transplantation. N Engl J Med. 2012; 366:586-589.

42. National Institute for Health and Care Excellence. Immunosuppressive therapy for renal transplantation in adults. 2004. Available from: http:// www.nice.org.uk/guidance/ta85. Accessed September 3, 2014.

43. Beckebaum S, Jacob S, Sweid D, et al. Efficacy, safety, and immunosuppressant adherence in stable liver transplant patients converted from a twice-daily tacrolimus-based regimen to once-daily tacrolimus extended-release formulation. Transpl Int. 2011;24:666-675.

44. Kuypers DR, Peeters PC, Sennesael JJ, et al. Improved adherence to tacrolimus once-daily formulation in renal recipients: a randomized controlled trial using electronic monitoring. Transplantation. 2013;95: 333-340.
Patient Preference and Adherence

\section{Publish your work in this journal}

Patient Preference and Adherence is an international, peer-reviewed, open access journal that focuses on the growing importance of patient preference and adherence throughout the therapeutic continuum. Patient satisfaction, acceptability, quality of life, compliance, persistence and their role in developing new therapeutic modalities and compounds to optimize

\section{Dovepress}

clinical outcomes for existing disease states are major areas of interest for the journal. This journal has been accepted for indexing on PubMed Central. The manuscript management system is completely online and includes a very quick and fair peer-review system, which is all easy to use. Visit http://www. dovepress.com/testimonials.php to read real quotes from published authors. 